\title{
Swinging-Up and Stabilization Control Based on Natural Frequency for Pendulum Systems
}

\author{
Noriko Matsuda, Masaki Izutsu, Jun Ishikawa, Katsuhisa Furuta and Karl J. Åström
}

\begin{abstract}
This paper proposes a swinging-up and stabilization method for inverted pendulums based on a pendulum oscillation model. A normalized oscillation model is derived by using only one model parameter, i.e., the natural frequency of a pendulum. As a control strategy, energy-based control with a normalized energy model is utilized for the swinging-up from the pendant position. For the stabilization of the pendulum at the upright position, variable structure system (VSS) type adaptive control is designed through the linearized oscillation model. Validity of the proposed method has been confirmed by simulation of an inverted pendulum on a cart. Swingingup and stabilization control was realized by using only the natural frequency of the pendulum, and the proposed method was also confirmed to be robust against parameter perturbation for the natural frequency. Stability of the designed controller was proven for a given condition that angular velocity of the pendulum has an upper bound.
\end{abstract}

\section{INTRODUCTION}

It is known that an inverted pendulum system, which is a typical underactuated mechanical system, is suitable for verifying new control theories. Usually control purpose of an inverted pendulum is to stabilize it at the upright position. For swinging-up control of the pendulum from the pendant position to the upright position, nonlinearity should be considered. To design controllers for swingingup and stabilization, a dynamic model of the pendulum is usually required, and it is necessary to know the model parameters. In designing advanced controllers, it is desirable to reduce the number of model parameters to be identified from a viewpoint of reducing affect of the identification and modeling errors. Adaptive control is known to be an alternative to solve the modeling error problem [1][2]. For the swinging-up of the inverted pendulum, energy-based control, which is one of the effective strategies to handle nonlinearities, was proposed by Astrom and Furuta [3], and many results for underactuated systems have been reported [4][5][6][7]. This method focuses on energy of the pendulum oscillation model. In [3], it is also shown that the oscillation model and energy of the pendulum are derived as functions

N. Matsuda is with the Department of Computer and Systems Engineering, Tokyo Denki University, Ishizaka, Hatoyama, Saitama 350-0394, Japan. matsuda@furutalab.k.dendai.ac.jp

M. Izutsu is with the Department of Advanced Multidisciplinary Engineering, Tokyo Denki University, Ishizaka, Hatoyama, Saitama 350-0394, Japan. idutsu@furutalab.k.dendai.ac.jp

J. Ishikawa is with Tokyo Denki University, 2-2 Nishiki, Kanda, Chiyoda, Tokyo 101-8457, Japan. ishikawa@fr.dendai.ac.jp

K. Furuta is with Tokyo Denki University, 2-2 Nishiki, Kanda, Chiyoda, Tokyo 101-8457, Japan. furuta@k. dendai.ac.jp

K. J. Åström is with the Department of Automatic Control LTH, Lund University, Box 118, SE-221 00 Lund, Sweden. karl_johan.astromecontrol.lth.se
TABLE I

PARAMETERS OF PENDULUM MODEL

\begin{tabular}{c|c|c}
\hline$m$ & {$[\mathrm{~kg}]$} & mass of pendulum \\
\hline$l$ & {$[\mathrm{~m}]$} & length from join to COG \\
\hline$J$ & {$\left[\mathrm{~kg} \cdot \mathrm{m}^{2}\right]$} & moment of inertia on joint \\
\hline$g$ & {$\left[\mathrm{rad} / \mathrm{s}^{2}\right]$} & gravity acceleration \\
\hline
\end{tabular}

of only the natural frequency. Hamiltonian approach and controlled Lagrangian method are similar to energy-based control.

In this paper, a swinging-up and stabilization method of inverted pendulums is proposed using the pendulum oscillation model. The pendulum's natural frequency can be easily derived from the pendulum oscillation period. This oscillation model is used to design controllers for both the swinging-up and stabilization of the pendulum. By using the pendulum oscillation model derived only from the natural frequency, the proposed method can stabilize the whole states of the pendulum system. The proposed method essentially uses two controllers. One is an energy-based controller using a normalized energy to swing up the pendulum. The other is a variable structure system (VSS) type adaptive controller for the linearized oscillation model to stabilize the pendulum system near the upright position. Stability of the proposed method is analyzed for the inverted pendulum on a cart, and the effectiveness is confirmed by simulation.

In Section II, a normalized oscillation model and an energy model are defined. An inverted pendulum system is modeled in Section III. Section IV proposes a controller design based on the oscillation model for swinging-up and stabilization. In Section V, the proposed controller design is validated by simulation. In Section VI, the stability of designed controllers is analyzed.

\section{NORMALIZED OSCILlation MODEL AND NORMALIZED ENERGY MODEL}

In this section a normalized oscillation model and a normalized energy model are defined by using the natural frequency of a pendulum. Figure 1 shows the pendulum model and Table I lists the model parameters.

The equation of motion of the pendulum-link shown in Fig. 1 is given by

$$
J \ddot{\theta}=m g l \sin \theta-m l u_{a} \cos \theta
$$

where $u_{a}$ is the input acceleration. Hereinafter, this equation of motion (1) is referred to the oscillation model of pendu- 


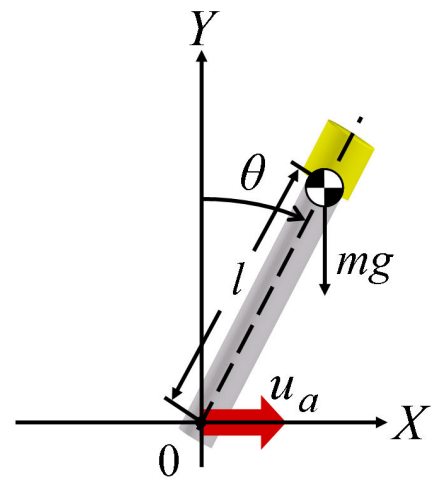

Fig. 1. Pendulum model

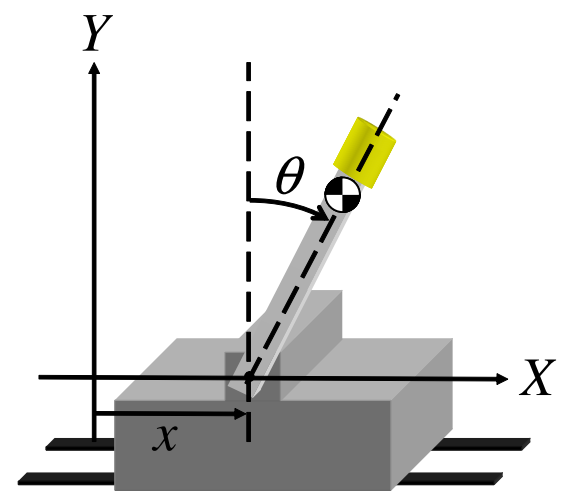

Fig. 2. Inverted pendulum on a cart

lum. The energy of the pendulum (1) is

$$
E=\frac{1}{2} J \dot{\theta}^{2}+m g l(\cos \theta-1) .
$$

Note that the potential energy becomes zero at the upright position $(\theta=0)$.

Divide (1) by $m g l$ to get the normalized oscillation model

$$
\frac{1}{\omega^{2}} \ddot{\theta}-\sin \theta=-\frac{1}{g} u_{a} \cos \theta .
$$

where $\omega=\sqrt{m g l / J}$ is the natural frequency of the pendulum. The normalized energy model of (3) is also given by

$$
E_{n}:=\frac{E}{m g l}=\frac{1}{2 \omega^{2}} \dot{\theta}^{2}+\cos \theta-1 .
$$

This paper presents a controller design method that is focused on the normalized pendulum model (3) and the normalized energy model (4).

\section{Model of Inverted Pendulum on a CART}

In this section, an equation of motion of an inverted pendulum on a cart is derived by using Euler-Lagrange method. The inverted pendulum on a cart is illustrated in Fig. 2. Table II lists the model parameters. Defining $q=[x, \theta]^{T}$, $\tau=[u, 0]^{T}$, the Lagrange equation is given by
TABLE II

PARAMETERS OF INVERTED PENDULUM ON THE CART

\begin{tabular}{c|c|c}
\hline$m$ & {$[\mathrm{~kg}]$} & mass of pendulum \\
\hline$l$ & {$[\mathrm{~m}]$} & length from joint to COG of pendulum \\
\hline$J$ & {$\left[\mathrm{~kg} \cdot \mathrm{m}^{2}\right]$} & moment of inertia on COG of pendulum \\
\hline$\theta$ & {$[\mathrm{rad}]$} & angle of pendulum \\
\hline$M$ & {$[\mathrm{~kg}]$} & mass of cart \\
\hline$x$ & {$[\mathrm{~m}]$} & displacement of cart \\
\hline$u$ & {$[\mathrm{~N}]$} & input to cart \\
\hline
\end{tabular}

Defining $q=[x, \theta]^{T}, \tau=[u, 0]^{T}$, the Lagrange equation is given by

$$
\frac{d}{d t} \frac{\partial L}{\partial \dot{q}}-\frac{\partial L}{\partial q}=\tau
$$

where $L(=T-U)$ is the Lagrangian, $T$ is summation of the kinetic energy of the cart and the pendulum, and $U$ is summation of the potential energy. Using (5), the equation of motion can be derived as follows:

$$
\begin{gathered}
M(\theta) \ddot{q}+h(\theta, \dot{\theta})+G(\theta)=\left[\begin{array}{l}
u \\
0
\end{array}\right] \\
M(\theta)=\left[\begin{array}{cc}
M+m & m l \cos \theta \\
m l \cos \theta & J
\end{array}\right] \\
h(\theta, \dot{\theta})=\left[\begin{array}{c}
-m l \dot{\theta}^{2} \sin \theta \\
0
\end{array}\right] \\
G(\theta)=\left[\begin{array}{c}
0 \\
-m g l \sin \theta
\end{array}\right]
\end{gathered}
$$

where $J\left(=\bar{J}+m l^{2}\right)$ is the moment of inertia around the pivot, $M(\theta)$ is the inertia matrix, $h(\theta, \dot{\theta})$ is the combination term of centrifugal, Coliolis and frictional forces and $G(\theta)$ is the gravity term.

If all the physical parameters are given, $\ddot{q}$ can be calculated by

$$
\ddot{q}=-M^{-1}(h+G)+M^{-1}\left[\begin{array}{l}
1 \\
0
\end{array}\right] u
$$

Hence, the acceleration of the cart $\ddot{x}$ and the input force to the cart $u$ satisfy the following relation:

$$
\ddot{x}=f_{0}+g_{0} u
$$

$$
\begin{aligned}
f_{0} & =-f_{N} / g_{D}, \quad g_{0}=J / g_{D} \\
f_{N} & =J m l \dot{\theta}^{2} \sin \theta-m^{2} g l^{2} \sin \theta \cos \theta \\
g_{D} & =J(M+m)-m^{2} l^{2} \cos ^{2} \theta .
\end{aligned}
$$

Note that $g_{0}>0$ for all $\theta, \dot{\theta}$. By deriving the nonlinear feedback law u rewriting (8), it is possible to directly design the acceleration input. This means that the controller can be designed from the normalized oscillation model (3). However, it is difficult to exactly identify all the parameters. Thus, this paper proposes a controller design method using only the natural frequency of pendulum. The following section gives a controller design using the normalized oscillation model. 


\section{Controller Design}

In this section, swinging up and stabilization controllers based on the normalized oscillation model and normalized energy model are designed. In the proposed design, around the upright position, a stabilization controller based on VSS type adaptive control is used, and in the other state apart from the upright position, a swinging-up controller is applied. Swinging-up and stabilization control are realized by switching the above two controllers. Switching condition is decided by the pendulum angle.

\section{A. Swinging-Up Controller}

From (3), the normalized oscillation model of the inverted pendulum on a cart can be described as

$$
\frac{1}{\omega^{2}} \ddot{\theta}-\sin \theta=-\frac{1}{g} \ddot{x} \cos \theta .
$$

where $\omega$ is the natural frequency. Similarly, the normalized energy model is given by

$$
E_{n}=\frac{1}{2 \omega^{2}} \dot{\theta}^{2}+\cos \theta-1 .
$$

From (9) and (10), the derivative of $E_{n}$ is

$$
\begin{aligned}
\dot{E}_{n} & =\left(\frac{1}{\omega^{2}} \ddot{\theta}-\sin \theta\right) \dot{\theta} \\
& =-\frac{1}{g} \dot{\theta} \ddot{x} \cos \theta .
\end{aligned}
$$

Consider the following Lyapunov function candidate (12) for the normalized energy

$$
V_{p}=\frac{1}{2}\left(E_{n 0}-E_{n}\right)^{2}
$$

where $E_{n 0}$ is the desired energy. From (11) and (12), we obtain

$$
\begin{aligned}
\dot{V}_{p} & =\frac{1}{g} \alpha \ddot{x} \\
\alpha & =\left(E_{n 0}-E_{n}\right) \dot{\theta} \cos \theta .
\end{aligned}
$$

Substituting (8) into (13), the derivative of $V$ is rewritten as

$$
\dot{V}_{p}=\frac{1}{g} \alpha\left(f_{0}+g_{0} u\right) \text {. }
$$

Thus, the control input is designed as

$$
u=-K \operatorname{sgn}(\alpha) \quad(K>0)
$$

where $\alpha$ is defined in (13) and $K$ is a gain. In the control law (15), the model parameter needed is only the natural frequency of the pendulum. Substituting (15) into (13), $\dot{V}_{p}$ becomes

$$
\dot{V}_{p}=\frac{1}{g} \alpha\left\{f_{0}-K g_{0} \operatorname{sgn}(\alpha)\right\} .
$$

In (15), if the gain $K$ satisfies the condition

$$
K \geq g_{0}^{-1} \operatorname{sgn}(\alpha) f_{0},
$$

$\dot{V}_{p}$ becomes semi-negative definite. Since the horizontal positions of the pendulum at $\theta= \pm \pi / 2$ are not an equilibrium point, $\cos \theta$ cannot be continuing to be zero. Then, the control law (15) drives the normalized energy $E_{n}$ to a desired value $E_{n 0}$, and this means that the pendulum is swung up.

\section{B. Stabilization Controller}

A stabilization controller based on VSS type adaptive control, which is proposed by Yamakita and Furuta [2], is applied for stabilizing the pendulum near the upright position.

1) Stabilization of Pendulum: When the pendulum energy is close to the desired energy by energy control, near the upright position, the angle and angular velocity of the pendulum $\theta, \dot{\theta}$ can be assumed to be small enough. The mass of cart is also assumed to be large enough, then the terms of the dynamics of the pendulum in (8) is regarded to be negligible small. Therefore, (8) is approximately rewritten as $\ddot{x}=u /(M+m)$. Under the assumptions, (9) is linearized at the upright position $(\theta=0, \dot{\theta}=0)$. Then we can obtain

$$
\ddot{\theta}=\omega^{2} \theta-\frac{1}{a} \omega^{2} u
$$

where $a=g(M+m)$. Define an estimation error $\tilde{a}$ by

$$
\tilde{a}=a-\hat{a}
$$

where $\hat{a}$ denotes the estimate of $a$. Reference value for the angle of the pendulum $\theta$ is denoted by $r$, then the tracking error $e$ is given by

$$
e=\theta-r \text {. }
$$

Define the switching function $s$ as

$$
s=\dot{e}+h e . \quad(h>0)
$$

Then, the time derivative of $s$ becomes

$$
\begin{aligned}
\dot{s} & =\ddot{\theta}-\ddot{\theta}_{r} \\
\ddot{\theta}_{r} & =\ddot{r}-h \dot{e} .
\end{aligned}
$$

Consider the following Lyapunov function candidate

$$
V_{u}=\frac{1}{2} a s^{2}+\frac{1}{2} \Gamma \tilde{a}^{2} . \quad(\Gamma>0)
$$

The time derivative of $V_{u}$ is given by

$$
\dot{V}_{u}=\omega^{2} s\left\{a\left(\theta-\frac{1}{\omega^{2}} \ddot{\theta}_{r}\right)-u\right\}+\Gamma \tilde{a} \dot{\tilde{a}} .
$$

Choose the control law as

$$
u=\hat{a}\left(\theta-\frac{1}{\omega^{2}} \ddot{\theta}_{r}\right)+\gamma \operatorname{sgn}(s) . \quad(\gamma>0)
$$

From (24) and (25), $\dot{V}_{u}$ is

$$
\dot{V}_{u}=-\gamma \omega^{2}|s|+\tilde{a}\left\{\omega^{2} s\left(\theta-\frac{1}{\omega^{2}} \ddot{\theta}_{r}\right)+\Gamma \dot{\tilde{a}}\right\} .
$$

The adaptive law that makes the second term of (26) to be zero is given by

$$
\dot{\hat{a}}=-\Gamma^{-1} \omega^{2} s\left(\theta-\frac{1}{\omega^{2}} \ddot{\theta}_{r}\right) .
$$

Then, $\dot{V}_{u}$ becomes

$$
\dot{V}_{u}=-\gamma \omega^{2}|s| \leq 0 .
$$

Consequently, the system states reach the sliding surface $(s=0)$ in finite time. The tracking error $e$ converges to zero because the sliding surface is stable. 
2) Design of Reference: The stabilization controller designed in the above section stabilizes only the pendulum angle, then the displacement of the cart should be controlled by another method. The reference $r$ for the pendulum angle that stabilizes the displacement and velocity of the cart should be designed. While the controller makes the pendulum angle track the reference $r$, the cart states can be simultaneously stabilized. In this section, stable reference angular acceleration $\ddot{r}$ that stabilizes the cart is designed. When states of the controlled system track the reference, (3) can be rewritten as

$$
\ddot{x}=-\frac{g}{\omega^{2}} \ddot{r}+g r
$$

where the gravity acceleration $g$ is assumed to be given. Defining the state vector $z=\left[\begin{array}{llll}x & \dot{x} & r & \dot{r}\end{array}\right]^{T}$, which is composed of the states of the cart and reference, the state equation is given by

$$
\dot{z}=A z+B \ddot{r}
$$

where

$$
A=\left[\begin{array}{llll}
0 & 1 & 0 & 0 \\
0 & 0 & g & 0 \\
0 & 0 & 0 & 1 \\
0 & 0 & 0 & 0
\end{array}\right], \quad B=\left[\begin{array}{c}
0 \\
-\frac{g}{\omega^{2}} \\
0 \\
1
\end{array}\right]
$$

Consider a quadratic criterion function

$$
J=\int_{0}^{\infty}\left(z^{T} Q z+R \ddot{r}^{2}\right) d t
$$

where $Q$ and $R$ are the weighting matrices. The optimal input $\ddot{r}$ minimizing (31) is given by

$$
\ddot{r}=-F z=-R^{-1} B^{T} P z
$$

where $F$ is a feedback gain vector. The matrix $P$ in (32) is the solution of the Riccati equation

$$
P A+A^{T} P-P B R^{-1} B^{T} P+Q=0 .
$$

\section{Switching Condition of Two Controllers}

The swinging-up and stabilization control is realized by switching the above two controllers depending on the pendulum angle. Because the VSS type adaptive controller is designed for the linearized model, the effective area in the phase plane is limited. On the other hand, energy control works for all the state range while it is difficult to stabilize the state. Thus, the switching condition is set as follows:

$$
u= \begin{cases}\hat{a}\left(\theta-\frac{1}{\omega^{2}} \ddot{\theta}_{r}\right)+\gamma \frac{s}{|s|+\mu} & \text { if }|\theta|<\theta_{s w} \\ -K \frac{L}{|L|+\epsilon} & \text { otherwise }\end{cases}
$$

where $\theta_{s w}$ are the switching angle. To eliminate the chattering, small constant values $\mu$ and $\epsilon$ are added to the control laws (15) and (25), respectively.

In the switching control law (34), only the pendulum's natural frequency $\omega$ is needed in designing the controllers.
TABLE III

SiMUlation PARAMETERS

\begin{tabular}{c|c|c||c|c|c}
\hline$m$ & {$[\mathrm{~kg}]$} & 0.2 & $J$ & {$\left[\mathrm{~kg} \cdot \mathrm{m}^{2}\right]$} & $2.67 \times 10^{-3}$ \\
\hline$l$ & {$[\mathrm{~m}]$} & 0.2 & $M$ & {$[\mathrm{~kg}]$} & 2.0 \\
\hline
\end{tabular}

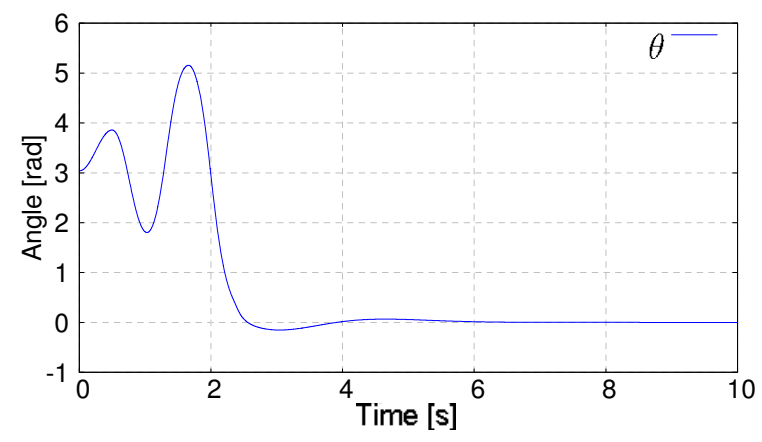

Fig. 3. Angle of pendulum : $\theta$

\section{Simulation}

The proposed controller is evaluated by simulation for an inverted pendulum on a cart. Furthermore, in order to verify a robustness for the parameter perturbation, this section considers the case of using the controller with different values from the true value of $\omega$. Simulation parameters of the inverted pendulum system are listed in Table III. The natural frequency $\omega$ of the pendulum for the parameters in Table III is $6.07[\mathrm{rad} / \mathrm{s}]$. The sampling interval is $2[\mathrm{~ms}]$ and the input force is limited within $\pm 10[\mathrm{~N}]$. The initial states are set to be $\left[\begin{array}{llll}x & \theta & \dot{x} & \dot{\theta}\end{array}\right]=\left[\begin{array}{llll}0 & \pi-0.1 & 0 & 0\end{array}\right]$. The design parameters are chosen as follows: $\theta_{s w}=\pi / 6, E_{n 0}=0, K=7, h=10$, $\Gamma=100, \gamma=10, \mu=\epsilon=0.05, Q=\operatorname{diag}(1,1,10,1)$, $R=1$. The stability analysis for the gain $K$ is discussed in Section VI. The initial condition of estimation value $\hat{a}$ is set to be $0.2 a$. It is assumed that all the states are assumed to be measured in this simulation.

\section{A. Nominal case}

This section shows the case of the controller using the true value of $\omega$.

Figs. 3 to 7 show the simulation results. From Fig. 3, the pendulum goes up to the upright position $(\theta=0[\mathrm{rad}])$. Fig. 4 shows that the normalized energy is pumped up until the reference value, and the normalized energy is settled into zero. Fig. 5 shows that the displacement of the cart converges to zero after the pendulum angle is stabilized. The control input becomes large when the pendulum swings up, and after the pendulum reaches the upright position, the control input converges to zero (Fig. 6). In Fig. 7, it is shown that the reference acceleration $\ddot{r}$ is generated from $2.2[\mathrm{sec}]$ after the stabilizing controller is activated. Then the generated reference acceleration $\ddot{r}$ converges to zero.

\section{B. Perturbed case}

To evaluate the proposed controller for parameter perturbation, we now consider the situation that is using $\hat{\omega}$ that is 


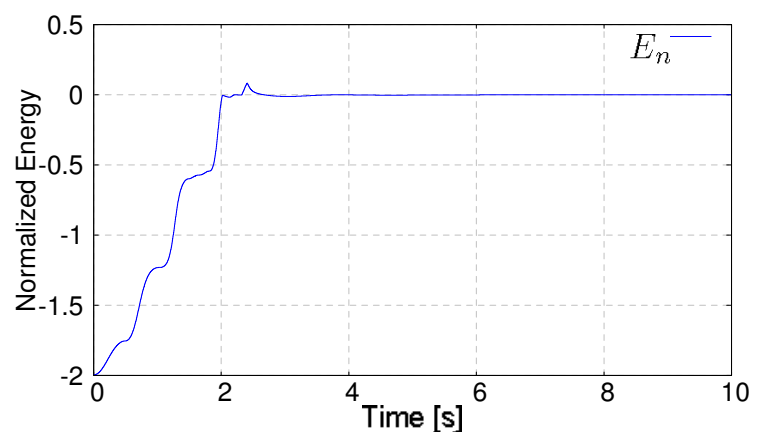

Fig. 4. Normalized energy : $E_{n}$

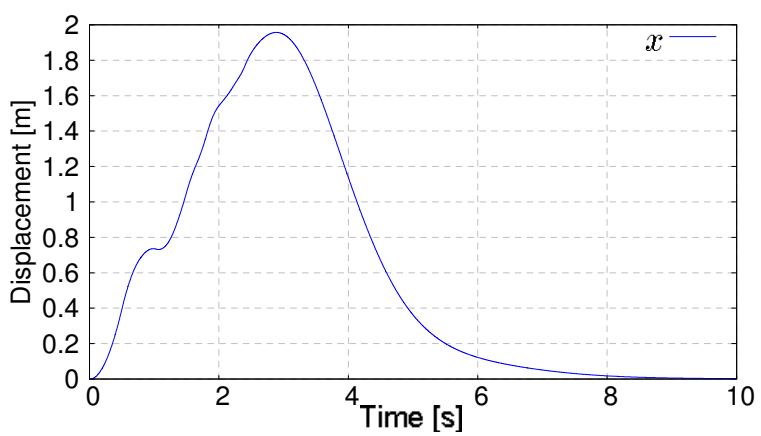

Fig. 5. Displacement of cart : $x$

different from the true value of $\omega$. If $\omega>\hat{\omega}$, the pendulum does not reach the upright position, because the calculated energy is larger than the true value of the energy. However, by setting the reference energy $E_{n 0}$ larger than the energy of the upright position with the velocity of zero, i.e., zero, the pendulum swings up until the upright position. On the other hand, if $\omega<\hat{\omega}$ and $E_{n 0}=0$, the calculated energy is smaller than the actual energy. Though the angular velocity is not zero, when the controller switches stabilization from swinging-up, the system states reach the stabilization area.

In this section, as a compromise the desired energy $E_{n 0}$ is set to be 5 , and the other design parameters are the same as those of the above simulation. Under the above condition, it is shown that swinging-up and stabilization control are achieved for the parameter variation of $\hat{\omega}$ from $0.5 \omega$ to $4 \omega$.

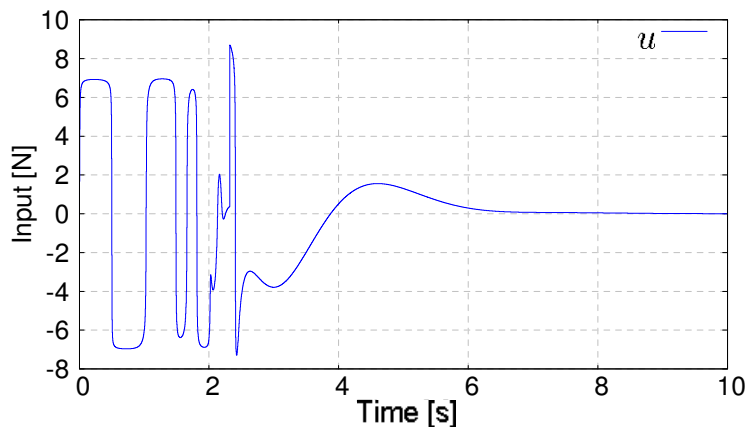

Fig. 6. Input : $u$

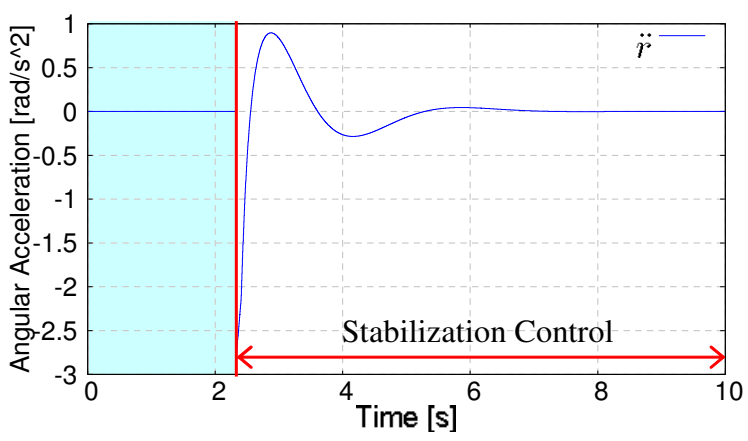

Fig. 7. Reference acceleration : $\ddot{r}$

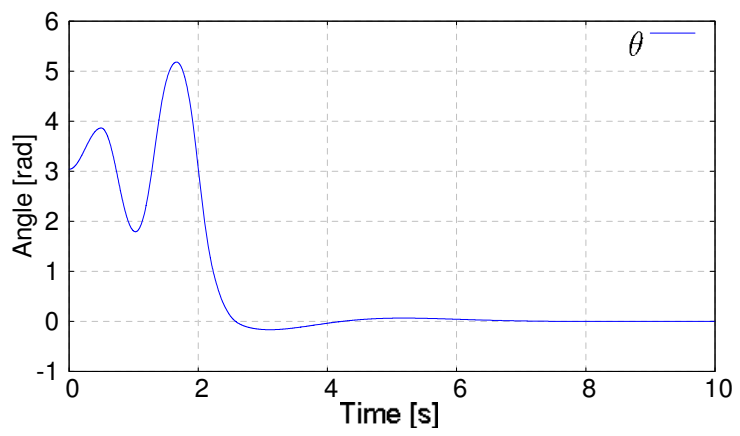

Fig. 8. Angle of pendulum : $\theta(\hat{\omega}=0.5 \omega)$

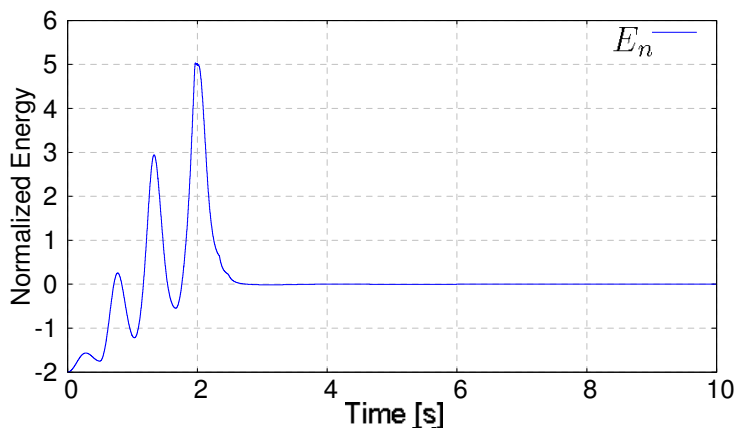

Fig. 9. Normalized energy : $E_{n}(\hat{\omega}=0.5 \omega)$

The simulation results for the smallest value $\hat{\omega}=0.5 \omega$ and the largest one $\hat{\omega}=4 \omega$ are shown in Figs. 8, 9 and Figs. 10, 11, respectively. From Fig. 8, the pendulum angle is confirmed to be settled into zero. In Fig. 9, it is found that the normalized energy goes up to the desired value 5, and after the pendulum stabilize at the upright position, the normalized energy converges to zero. From Figs. 10 and 11 , for $\hat{\omega}=4 \omega$ the pendulum angle and the normalized energy is also stabilized. We also confirmed that our method successfully works for other value of $\hat{\omega}$ from $0.5 \omega$ to $4 \omega$. Due to the limitation of the space, however, these details in other parameters are omitted.

Consequently, the proposed control designed by using only the natural frequency has achieved both the swinging-up and stabilization of the inverted pendulum system. 


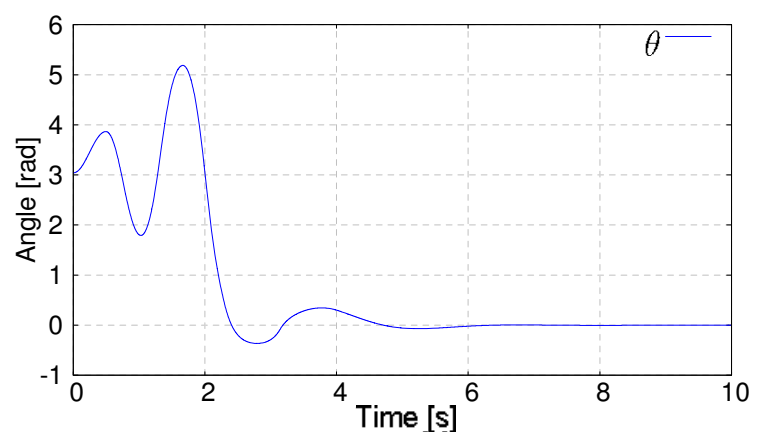

Fig. 10. Angle of Pendulum : $\theta(\hat{\omega}=4 \omega)$

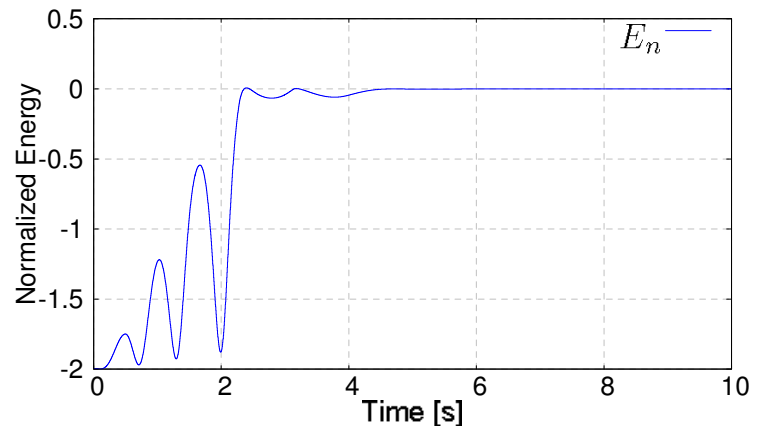

Fig. 11. Normalized energy : $E_{n}(\hat{\omega}=4 \omega)$

\section{AnAlysis of Swinging-Up Controller}

In Section VI, it is proven that the normalized energy $E_{n}$ can be converged to the desired value $E_{n 0}$ if the gain $K$ satisfies the condition $K \geq g_{0}^{-1} \operatorname{sgn}(\alpha) f_{0}$. To show the existence of $\mathrm{K}$ which satifies (17), the maximum value of $g_{0}^{-1} \operatorname{sgn}(\alpha) f_{0}$ is calculated. The model parameters used here are the same as those of the above simulation.

Define the following function

$$
f(\theta, \dot{\theta}):=g_{0}^{-1} \operatorname{sgn}(\alpha) f_{0} .
$$

Consider the case that the pendulum is swung up from the pendant position to the upright position by pumping the energy. When the normalized energy $E_{n}$ converges to the desired value $E_{n 0}$, the absolute value of the angular velocity of the pendulum is calculated as

$$
|\dot{\theta}|=\omega \sqrt{2\left(E_{n 0}-\cos \theta+1\right)}
$$

From (36), the maximum amplitude of angular velocity is $12.13[\mathrm{rad} / \mathrm{s}]$ for $E_{n 0}=0$ and $\theta=\pi$. Therefore, it can be considered that the function $f$ varies within a range calculated from $\theta \in[-\pi, \pi], \dot{\theta} \in[-13,13]$. The time derivative of $\theta_{m}$ as a function of the angular velocity that maximizes the function $f$ is given by

$$
\dot{\theta}_{m}(\theta)=\arg \max _{\dot{\theta}}\{f(\theta, \dot{\theta})\} .
$$

Fig. 12 shows $\dot{\theta}_{m}(\theta)$ as a function of the pendulum angle $\theta$ varying from $-\pi$ to $\pi$.

Fig. 13 shows $f\left(\theta, \dot{\theta}_{m}(\theta)\right)$ derived from (37). From Fig. 13 , the maximum value of $g_{0}^{-1} \operatorname{sgn}(\alpha) f_{0}$ is 6.91. Thus, if

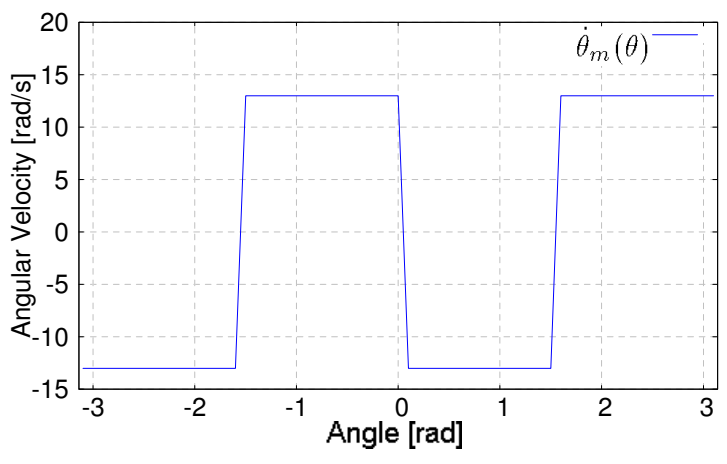

Fig. 12. Value of $\dot{\theta}_{m}(\theta)$

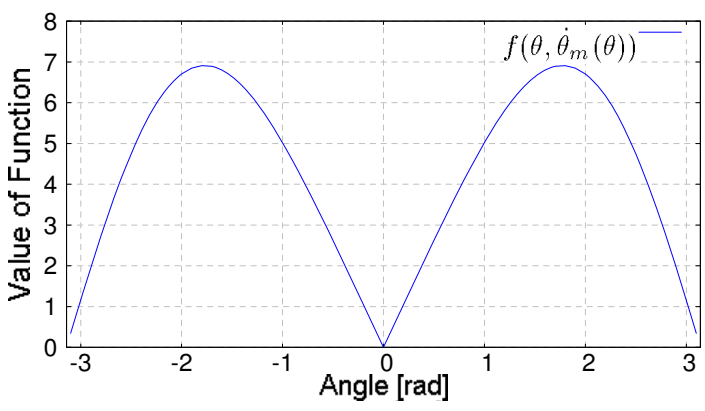

Fig. 13. Value of $f\left(\theta, \dot{\theta}_{m}(\theta)\right)$

$K \geq 6.91$, the normalized energy converges the desired value.

\section{CONCLUSION}

A normalized oscillation model and an energy model that are characterized by the natural frequency of the pendulum were derived. A swinging-up controller based on the normalized energy model was proposed for pendulum systems, and stability of the control system was proven by numerical analysis. Efficiency of the proposed method was shown by simulation for an inverted pendulum on a cart. Additionally, robustness for perturbation of the natural frequency was also confirmed.

\section{REFERENCES}

[1] J.-J. E. Slotine, W. Li, "On the Adaptive Control of Robot Manipulators," International Journal of Robotics Research, vol. 6, no. 3, 1987, pp. $49-59$.

[2] M. Yamakita, K. Furuta, "VSS Adaptive Control Based on Nonlinear Model for TITech Pendulum," IEEE, 1992, pp. 1488-1493.

[3] K. J. Åström, K. Furuta, "Swinging Up a Pendulum by Energy Control," Automatica, vol. 36, no. 2, 2000, pp. 287-295.

[4] M. Iwashiro, K. Furuta, K. J. Åström, "Energy Based Control of Pendulum," Proceedings of IEEE Conference on Control Applications, 1996, pp. 715-720.

[5] K. J. Åström, K. Furuta, M. Iwashiro, T. Hoshino, "Energy Based Strategies for Swinging up a Double Pendulum," Proceedings of the 14th IFAC World Congress, 1999, pp. 283-288.

[6] X. Xin, M. Kaneda, "Analysis of the Energy-Based Control for Swinging Up Two Pendulums," IEEE Trans. on Automatic Control, vol. 50, no. 5, 2005, pp. 679-684.

[7] T. Henmi, M. Deng, A. Inoue, N. Ueki, Y. Hirashima, "Swing-up Control of a Serial Double Inverted Pendulum," Proceeding of the 2004 American Control Conference, 2004, pp. 3992-3997. 